\title{
A STUDY OF THE PATIENTS ATTENDING THE ASTHMA ROOM AT GROOTE SCHUUR HOSPITAL
}

\author{
Renè Meyers, B.Sc. (Physiotherapy), U.C.T.*
}

On reviewing the literature on asthmatic patients, a general problem of patient education became evident (Westerman et al., 1978; Prinsloo, 1978). Groote Schuur Hospital was thought to be an ideal place to study such patients and find out just how much they do know.

The Asthma Room is part of the Medical Casualty Department and serves as a short stay ward for patients with acute attacks of asthma. The room is supplied with piped oxygen which is used to administer nebulized bronchodilators. The patients are accommodated in arm chairs.

According to the Emergency Unit register, 1005 patients were admitted to the Asthma Room over a 10-month period from October 1977 to August 1978 (Prinsloo, 1978). The average number of admissions per patient was also extremely high. This study, by means of a questionnaire (Fig. 1), interviewed 82 patients in the Asthma Room during the period 2-27 April 1979.

The questions covered aspects of the patients' condition, and frequency of attacks. There were questions on the knowledge of their medication and physiotherapy techniques. A demonstration of the use of the inhaler by the patient was required. Broader questions were included to give the patient the opportunity to advise fellow-asthmatics and to gauge their opinion of the atmosphere in the Asthma Room. Some of the results obtained are discussed below.

The total number of admissions of the 82 patients studied, amounted to 801 . The majority of the patients had very little insight into the "mechanics of asthma". Most explained their condition as being tightness of the chest or shortness of breath. Only $9,8 \%$ mentioned bronchi or "lung pipes" and none of these could expand any further. Although a high percentage felt they knew; and stated, the trigger factor for the present attack, the majority did not seem to associate avoiding the trigger factor with preventing an attack.

Over $75 \%$ of the patients were breathing apically. Only $37 \%$ were aware that their breathing pattern could be more effective. Those patients who knew how to breathe diaphragmatically, and realized its importance, had previously been admitted to another ward where they had received physiotherapy treatment and thus breathing reeducation. Only 7,3\% used physiotherapy techniques such as relaxation, resting positions and

* Physiotherapist, Groote Schuur Hospital, Cape Town.

$\uparrow$ Summary of undergraduate project presented towards B.Sc, (Physiotherapy) in 1979. breathing control, to prevent the present attack from becoming worse.

Medication proved to be a problem. All but 3,7\% found that the medication required was easily obtainable, and the place from which they received it, was easily accessible. Yet at the time of admission, $46 \%$ had "run out" of some of their medication and $16 \%$ had no medicine left at all. A demonstration by the patients on the use of the inhaler revealed that $40 \%$ used their inhaler effectively. Many had been using an inhaler for several years. Judging from the demonstration, they had probably never been taught how to use it and did not benefit fully from the inhalation.

A large variety of drugs were prescribed for these patients with an average of 3 per patient. As many of the patients interviewed did not understand the value of prophylactic medicine, it was clear that more time should be spent explaining the dosage of each drug and importance of regular and correct use. During the interview patients were encouraged to express their views and thought they could help other asthma patients by advice mainly on home remedies.

The results obtained reinforced the original hypothesis, namely that if the patient had a better knowledge of their disease and of their particular trigger factors, they could avoid precipitating attacks. With the added knowledge of physiotherapy techniques and the correct usage of medication, they could institute immediate and more effective "self-treatment" at home. In this way they should be able to abort an attack or prevent it from reaching the stage where professional help is required.

The Asthma Room would be an ideal place to institute a group education programme, including informal discussions with the physiotherapist. A more meaningful rôle for physiotherapists in the education of all asthma patients could evolve and cover aspects such as resting positions, breathing control, relaxation. and the correct use of medication, especially inhalers. This could greatly assist patients to a better understanding of their disease as a whole.

\section{References}

1. Prinsloo, F. R. (1978). Department of Community Medicine, University of Cape Town (persona assignment).

2. Westermann, D. E., Bateman, E. D., Alexander, M. G., Benatar, S. R. (1978). The magnitude of the asthma problem in Cape Town. S.A.Med. J., 53 $544-547$. 